\title{
Guest editorial: efficacy of and resistance to molecularly targeted therapy for myeloid malignancies
}

\author{
Hitoshi Kiyoi
}

Received: 1 May 2013/Revised: 6 May 2013/Accepted: 6 May 2013/Published online: 12 May 2013

(C) The Japanese Society of Hematology 2013

The introduction of all-trans retinoic acid (ATRA) and the ABL kinase inhibitors has dramatically improved the prognosis of patients with acute promyelocytic leukemia (APL) and chronic myeloid leukemia (CML), respectively. These remarkable clinical successes have proved the principle of molecularly targeted therapy, raising expectations that therapeutic interventions targeting altered molecules will improve outcomes in patients with cancers and hematologic malignancies. A number of targeted agents, such as small molecular compounds and monoclonal antibodies, have already been approved and evaluated in clinical development; however, the accumulation of clinical information has also revealed cases of treatment failure due to resistance. Several mechanisms of resistance to targeted agents, such as mutation or amplification of the targeted gene, up-regulation of P-glycoprotein, activation of other pathways, and pharmacokinetic variability, have been observed in the clinical setting. The present issue of IJH includes four review articles focusing on the efficacy of and resistance to molecularly targeted therapy for myeloid malignancies.

Myeloproliferative neoplasms (MPN) are clonal hematopoietic stem cell disorders characterized by proliferation of one or more of the myeloid lineages. Although the diagnosis of CML is confirmed by the presence of the Philadelphia chromosome and/or $B C R-A B L 1$ fusion gene, other MPN subtypes are generally diagnosed based on their clinical and laboratory features. With the identification of the $J A K 2$ mutation in nearly all patients with polycythemia

H. Kiyoi $(\square)$

Department of Hematology and Oncology, Nagoya University

Graduate School of Medicine, Tsurumai-cho 65, Showa-ku,

Nagoya 466-8550, Japan

e-mail: kiyoi@med.nagoya-u.ac.jp vera (PV) and about half of those with essential thrombocythemia (ET) and primary myelofibrosis (PMF), the mutational status of the JAK2 gene is now considered among the diagnostic criteria for PV, ET, and PMF. Mutant JAK2 is constitutively activated and introduces the activation of downstream molecules, such as the STAT, MAPK, and PI3K pathways, promoting the transformation and proliferation of hematopoietic progenitors. Several JAK2 inhibitors, therefore, have been evaluated in clinical development. Neha Bhagwat, Ross L. Levine, and Priya Koppikar summarize recent advances in the development of JAK2 inhibitors, and clearly show that chronic JAK inhibition leads to JAK inhibitor persistence via transphosphorylation of JAK2 through other JAK family members, a crucial observation for the development of therapeutic strategies using JAK inhibitors in MPN.

The FLT3 protein is a member of the class III receptor tyrosine kinases (RTK), which also includes KIT, FMS and PDGFR. Mutations in FLT3 represent the most frequently reported genetic alteration in AML, and are involved in the signaling pathway associated with autonomous proliferation and differentiation block in leukemia cells. The FLT3ITD mutation is strongly associated with leukocytosis and poor prognosis. The development of FLT3 kinase inhibitors may thus yield more efficacious strategies for leukemia therapy. Numerous FLT3 inhibitors have been tested in phase 1 and 2 clinical trials, but their efficacy in the treatment of AML has been unimpressive, and have been plagued by adverse effects and the development of resistance. In their review, Michael R. Grunwald and Mark J. Levis summarize the clinical efficacy of FLT3 inhibitors. They also clearly show several resistance mechanisms, as revealed by clinical trials, and suggest new treatment strategies in combination with FLT3 inhibitors for AML patients harboring FLT3 mutations. 
Even with intensive chemotherapy and/or allogeneic hematopoietic stem cell transplantation, the complete remission and overall survival rates in adult AML, with the exception of APL, remain about 80 and $40 \%$, respectively. Several small molecule compounds targeting altered molecules in AML have been developed, but efforts to develop them for clinical use remain unsuccessful. Gemtuzumab ozogamicin (GO) is a humanized anti-CD33 monoclonal antibody conjugated with calicheamicin. Although GO was initially approved by the Food and Drug Administration (FDA) in the United States for single-agent treatment of patients older than 60 years with AML, it was voluntarily withdrawn based on the results of a randomized phase III study by the Southwest Oncology Group (SWOG), S0106, in which the addition of GO to standard induction therapy did not provide clinical benefit for adult AML patients under 60 years of age. However, subsequent randomized studies strongly suggested the efficacy of GO with acceptable toxicity in newly diagnosed AML, highlighting the need to re-evaluate combination therapy with $\mathrm{GO}$ for
AML. Akihiro Takeshita comprehensively reviews the clinical efficacy of GO both as a monotherapy and a combination therapy. He further clearly summarizes the resistance mechanism of GO, which will need to be considered for any future strategy of GO-combination chemotherapy.

Finally, Akihiro Tomita, Hitoshi Kiyoi, and Tomoki Naoe summarize the mechanisms of action of ATRA and arsenic trioxide (ATO) in APL. They also clearly describe their mechanisms of resistance, which are apparent in relapsed and/or refractory patients. Although the prognosis of APL patients has been dramatically improved by the introduction of ATRA and ATO, this review indicates that further studies are required to obtain a cure of APL.

I hope that all readers will gain a fuller understanding of the state of the art in molecularly targeted therapy for myeloid malignancies from these excellent review articles, which also provide us with future directions for the development of therapeutic strategies to improve prognosis in patients with myeloid malignancies. 